\title{
GROWTH FUNCTIONS FOR SOME NONAUTOMATIC BAUMSLAG-SOLITAR GROUPS
}

\author{
MARCUS BRAZIL
}

\begin{abstract}
The growth function of a group is a generating function whose coefficients $a_{n}$ are the number of elements in the group whose minimum length as a word in the generators is $n$. In this paper we use finite state automata to investigate the growth function for the Baumslag-Solitar group of the form $\left\langle a, b \mid a^{-1} b a=a^{2}\right\rangle$ based on an analysis of its combinatorial and geometric structure. In particular, we obtain a set of length-minimal normal forms for the group which, although it does not form the language of a finite state automata, is nevertheless built up in a sufficiently coherent way that the growth function can be shown to be rational. The rationality of the growth function of this group is particularly interesting as it is known not to be synchronously automatic.

The results in this paper generalize to the groups $\left\langle a, b \mid a^{-1} b a=a^{m}\right\rangle$ for all positive integers $m$.
\end{abstract}

\section{INTRODUCTION}

Let $G$ be a group generated as a monoid by the finite set $X$; that is, every element of $G$ can be written as a word formed from $X$ without using inverses. Throughout this paper we will take $X$ to be $V \cup V^{-1}$, where $V$ is a finite set generating $G$ as a group. Let $w$ be a word in $X$. We denote the group element corresponding to $w$ as $\bar{w}$, and we define the length of $w, l(w)$, as the word length of $w$ with respect to $X$. For any $g \in G$, we define the length $l(g)$ of $g$ to be the minimum length of all words in $X$ representing $g$; so

$$
l(g)=\min \{l(w) \mid \bar{w}=g\} .
$$

In geometric terms, $l(g)$ is the length of the shortest path from the identity element to $g$ in the group graph of $G$ with respect to $X$, where each edge is assumed to have length 1 . Let $a_{n}:=\operatorname{card}\{g \in G \mid l(g)=n\}$. Then the growth series $\Gamma(G, X)(x)$ of $G$ is defined to be the usual generating function for the sequence $a_{n}$ :

$$
\Gamma(G, X)(x)=\sum_{n=0}^{\infty} a_{n} x^{n} .
$$

A group $G$ is said to have rational growth if the function $\Gamma(G, X)(x)$ is a rational function of $x$ for some choice of $X$. This is equivalent to saying that the $a_{n}$ 's satisfy some recurrence relation.

Received by the editors July 2, 1991.

1991 Mathematics Subject Classification. Primary 20F05; Secondary 20F10. 
Also, throughout this paper we will denote by $\mathbf{N}$ the set of natural numbers $\{1,2,3, \ldots\}$, and by $\mathbf{N}_{0}$ the set $\{0,1,2,3, \ldots\}$.

The class of groups with presentation $\left\langle a, t \mid t a^{p}=a^{q} t\right\rangle, p, q \in \mathbf{N}$, are often referred to as the Baumslag-Solitar groups (after Baumslag and Solitar's classification of when these group groups are Hopfian in [1]). The Baumslag-Solitar groups for which $p=q$ have been shown to be (synchronously) automatic; however, the groups for which $p \neq q$ are not synchronously automatic, or indeed even combable, but are asynchronously automatic, that is, have an automatic structure based on two tape automata. Details of these results can be found in $[2,6]$. Edjvet and Johnson [5] have shown that the Baumslag-Solitar groups with $p=q$ have rational growth. In this paper we will show that the group $G_{2}=\left\langle a, t \mid t a^{2}=a t\right\rangle$ also has rational growth, with respect to the symmetric generating set $X=\left\{a^{-1}, t^{-1}, a, t\right\}$. This appears to be the first known example of a nonautomatic group with rational growth. We will also briefly indicate how this result can be generalized to all groups $G_{p}=\left\langle a, t \mid t a^{p}=a t\right\rangle$.

A basic method of showing a group has rational growth is to find a regular set $S$ of length-minimal normal forms for the group (by 'regular' we mean that $S$ is the language of a finite state automaton). That the existence of such a set implies rational growth follows from the following theorem.

Theorem 1.1. Let $G$ be a group generated as a monoid by the finite set $X$. Let $S$ be a regular set of length-minimal normal forms for $G$ with respect to $X$. Then the growth function $\Gamma(G, X)(t)$ is a rational function of $t$.

The proof of this theorem (which can be found in [3]) is a simple application of the fact that the adjacency matrix of the finite state automaton satisfies its own characteristic equation. The result is a generalization of a theorem of Ghys and de la Harpe [7, Chapter 9, 2.14].

The strategy of this paper is to apply this theorem to the vertices of a particular subgraph of the Cayley graph of $G_{2}$, and then show that geodesics in the Cayley graph can be expressed in terms of paths in this subgraph. In $\S 2$ we specify this particular subset of $G_{2}$ and show that it has a set of length-minimal normal forms which is regular. Then, in $\S 3$ we use these normal forms to find length-minimal normal forms for the entire group.

The motivation for these normal forms comes from the geometry of the Cayley graph of $G_{2}$. So we will first describe how to form a picture of the group. The Cayley graph can be thought of as a directed graph with each edge labelled $a$ or $t$. We think of all the edges labelled $a$ as running horizontally from left to right, and all the edges labelled $t$ as running vertically from top to bottom. The graph is built out of closed circuits, each corresponding to the group's relation, $t a^{2}=a t$. Each of these circuits has one edge labelled $a$ running across the top and two consecutive edges labelled $a$ running across the bottom. We can form an infinite horizontal strip of these circuits by demanding each edge $t$ be shared by two consecutive circuits. Now each of the vertices along the top of this strip must have an edge labelled $t$ running into it. In order for this to occur there must be two more such horizontal strips attached above this one, positioned so that an edge labelled $t$ meets each vertex from above, as shown in Figure 1. In this way we build up a picture of the entire group as a (vertical) binary tree whose branches are built from these horizontal 


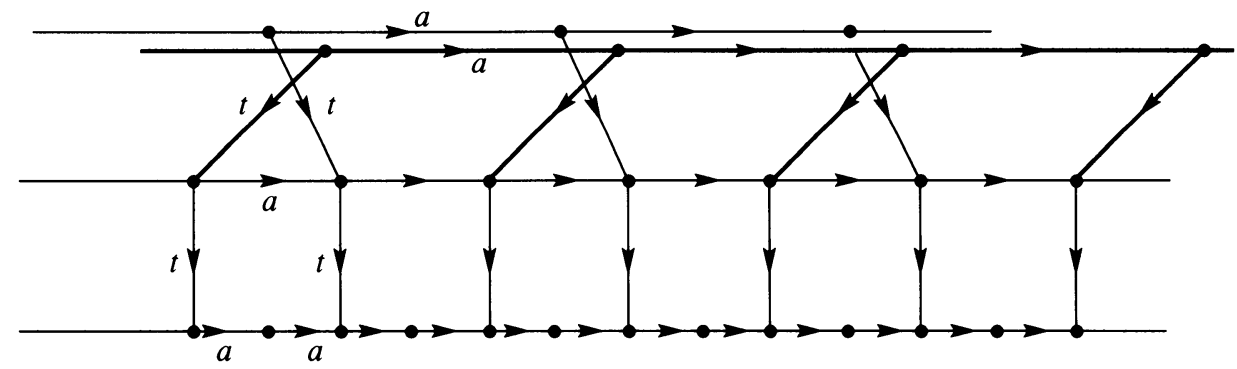

Figure 1. Part of the Cayley graph of $G_{2}$

strips. It will be helpful to keep this picture in mind throughout the following two sections.

\section{A REGULAR SET OF LENGTH-MINIMAL NORMAL FORMS}

Throughout this paper we will be investigating the group $G_{2}=\langle a, t| t a^{2}=$ at $)$. Note that $G_{2}$ is generated as a monoid by the set $X=\left\{a^{-1}, t^{-1}, a, t\right\}$. Our general aim is to find a way of describing length-minimal normal forms for the elements of this group, and show that these imply that the group grows rationally. In this section we will show that a particular subset of $G_{2}$ has a set of length-minimal normal forms which is regular, that is, is the language of a finite state automaton.

Before stating our main theorem for this section, we need to define some partial orderings for $G_{2}$. Let $x, y \in G_{2}$. We say that $x \succeq y$ if $y=x t^{m} a^{n}$ for some $m, n \in \mathbf{N}_{0}$; and that $x \succ y$ if $n>0$. Similarly we say that $y \preceq x$ if $y=x t^{m} a^{-n}$ for some $m, n \in \mathbf{N}_{0}$; and that $y \prec x$ if $n>0$. It will also be useful to define a more restricted partial ordering. We write that $x \backslash y$, if $x \succeq y$ but $y \prec x a$; and similarly that $y \swarrow x$ if $y \prec x$ but $x a^{-1} \succeq y$.

When considering words in $X$ the following definition will be useful. Let $v$ and $w$ be words in $X$. We say that $v$ is a head of $w$ if $w=v w_{0}$ for some $w_{0}$ in $X$, and similarly that $v$ is a tail of $w$ if $w=w_{0} v$ for some word $w_{0}$ in $X$.

Theorem 2.1. Let $S=\left\{g \in G_{2} \mid e \searrow g\right\}$. Then there is a set $M(S)$ of lengthminimal normal forms for elements of $S$, such that $M(S)$ is regular.

We chose $M(S)$ so that, under the ordering $t^{-1}<a^{-1}<t<a, M(S)$ is the set of lexicographically smallest length-minimal normal forms for $S$. We will show that this set is regular via a sequence of short lemmas each of which tell us something about the form of elements in $M(S)$.

The first lemma, whose proof is trivial, constitutes an important combinatorial tool which will be used repeatedly throughout this paper.

Lemma 2.2. Let $w$ be a word in $X$ such that $\bar{w}=a^{k}$ for some $k \in Z$. Then $\overline{a^{l} w}=\overline{w a^{l}}$ for all $l \in \boldsymbol{Z}$. 
Lemma 2.3. Let $w$ be a length-minimal word in $X$ such that

(i) either $t$ or at is a head of $w$, and

(ii) either $\bar{w} \prec e$ or $e \succeq \bar{w}$.

Then $w$ does not contain the letter $t^{-1}$ or any subword of the form $a^{ \pm m}$ where $m>1$.

Proof. Assume $w$ contains the letter $t^{-1}$. Then we can write $w$ in the form $w_{0} t a^{l} t^{-1} w_{1}$ where $w_{0}$ and $w_{1}$ are words in $X$ such that $w_{0}$ does not contain $t^{-1}$, and $l$ is an integer. We consider separately the cases where $l$ is even and $l$ is odd. First, assume $l=2 k$ for some $k \in Z$. In this case $\overline{t a^{2 k} t^{-1}}=\overline{a^{k}}$, and hence $t a^{2 k} t^{-1}$ is not length-minimal, giving a contradiction.

Alternatively, assume $l$ is odd. Since $\bar{w} \prec e$ or $e \succeq \bar{w}$, we can write $w$ as $w_{0} t a^{l} t^{-1} v_{0} v_{1}$ where $\overline{t^{-1} v_{0}}=a^{m}$ for some $m \in \mathbf{Z}$. So, by Lemma 2.2,

$$
\bar{w}=\overline{w_{0} t t^{-1} v_{0} a^{l} v_{1}} \text {. }
$$

But, clearly, $l\left(w_{0} t a^{l} t^{-1} w_{1}\right)=l\left(w_{0} t t^{-1} v_{0} a^{l} v_{1}\right)$, while $w_{0} t t^{-1} v_{0} a^{l} v_{1}$ is not length-minimal since it is not reduced, contradicting the assumption that $w$ is length-minimal.

For the second part of the lemma, assume $w$ contains a subword of the form $a^{ \pm m}$. Then it follows that $t a^{ \pm 2}$ is a subword of $w$. But $\overline{t a^{2}}=\overline{a t}$ and $\overline{t a^{-2}}=\overline{a^{-1} t}$ hence $t a^{ \pm 2}$ is not length-minimal.

Lemma 2.4. Let $w \in M(S)$, and let $v$ be a tail of $w$ such that

(i) $v$ does not contain the subwords $t^{-1}, t^{2}$, or $t a^{-2}$, and

(ii) $t$ is the first letter of $v$.

Then either $e \searrow \bar{v}$ or $\bar{v} \swarrow e$.

Proof. It follows from conditions (i) and (ii) that $v$ can be written in the form $v=u_{1} u_{2} \cdots u_{k}$ where each $u_{i} \in\left\{t, t a^{-1}, t a\right\}$. But if $a^{-1} \succ \overline{v_{0}}$ then $a^{-1} \succ$ $\overline{v_{0} u_{i}}$ for any $u_{i}$ in the above set. Similarly, if $\overline{v_{0}} \prec a$ then $\overline{v_{0} u_{i}} \prec a$. Hence $a^{-1} \succ \bar{v}$ and $\bar{v} \prec a$.

It will be useful in the following lemmas to consider a slightly larger set than $S$. Let $S^{\prime}=S \cup\left\{a t^{k} \mid k \in \mathbf{N}_{0}\right\}$, and let $M\left(S^{\prime}\right)$ be the set of lexicographically smallest length-minimal normal forms for $S^{\prime}$.

Lemma 2.5. Let $w \in M(S)$, and let $v$ be a head of $w$. Then $v \in M\left(S^{\prime}\right)$.

Proof. Let $g=\bar{w}$ and assume that $w$ is not the empty word. We will first show that either $t$ or at is a head of $w$. Let $w=w_{0} t w_{1}$ where $w_{0}$ is a word in $X$ not containing the letter $t$. We wish to show that $w_{0}$ is either $a$ or the empty word. First suppose that $w_{0}$ does not contain $t^{-1}$; that is, $w_{0}=a^{p}$ for some $p \in \mathbf{Z}$. But in this case either $g \prec \overline{w_{0}}$ or $\overline{w_{0}} \succeq g$. So, by Lemma 2.3, $w_{1}$ does not contain the subwords $t^{-1}, t a^{2}$ or $t a^{-2}$, and hence, by Lemma 2.4, $g \swarrow \overline{w_{0}}$ or $\overline{w_{0}} \searrow g$. This implies $p=0$ or 1 as desired.

Now assume $w_{0}$ contains the letter $t^{-1}$. We will show this leads to a contradiction. Since $e \succeq g$, we can write $w$ as $w=w_{0} t v_{0} v_{1}$ where $v_{0} v_{1}=w_{1}$ and $\overline{w_{0} t v_{0}}=a^{p}$ for some $p \in \mathbf{Z}$. Again by the above argument we can assume that $p=0$ or 1 , which contradicts the assumption the $w_{0} t v_{0}$ is length-minimal. So, either $t$ or at is a head of $w$.

The consequence of this is that Lemma 2.3 applies to every $w \in M(S)$, and that Lemma 2.4 applies to any tail of $w$ beginning with $t$. Now let $v$ be a 
head of $w$, so $w=v w_{2}$ for some length-minimal word in $X, w_{2}$. If $\bar{v} \in S^{\prime}$ then clearly $v \in M\left(S^{\prime}\right)$. If $v \notin S$ then, by Lemma 2.4 , either $\bar{v} \swarrow e$ or $a \searrow \bar{v}$. Assume $\bar{v} \swarrow e$. If $t$ is the first letter of $w_{2}$ then, again by Lemma 2.4, $\overline{w_{2}} \prec \overline{v a}$, and hence $g \prec e$ which is a contradiction to the assumption that $g$ is an element of $M\left(S^{\prime}\right)$. If $a$ is the first letter of $w_{2}$ then we can write $w_{2}=a w_{3}$ such that $t$ is the first letter of $w_{3}$. Either $\overline{v a}=t^{n}$ for some $n \in \mathbf{N}$, in which case $v a$ is not length-minimal, or $\overline{v a} \swarrow e$, in which case we get the same contradiction as above. The same contradiction also occurs if $a^{-1}$ is the first letter of $w_{2}$.

A similar argument shows there is also no suitable $v$ for which $a \searrow \bar{v}$, completing the proof.

Before stating the next lemma it will be useful first to define the length of elements or words in the group with respect to $t$. This concept will also be used extensively in $\S 3$. Let $g \in G_{2}$ such that $g=t^{k} a^{l}$ with $k \geq 0$. Then we call $k$ the t-length of $g$, written $l_{t}(g)$. Similarly, if $w$ is a word in $X$ such that $\bar{w}=t^{k} a^{l}$ with $k \geq 0$, then $l_{t}(w)=k$.

Lemma 2.6. Let $g$, $g a \in S^{\prime}$ such that $l(g)=l(g a)$, and let $w_{1}, w_{2} \in M\left(S^{\prime}\right)$ such that $\overline{w_{1}}=g$ and $\overline{w_{2}}=g a^{n}$, where $n>0$. Then, under the lexicographical ordering, $w_{1}<w_{2}$.

Proof. Let $v_{1}, u_{1} \in M\left(S^{\prime}\right)$ and let $\overline{v_{1}}=g_{1}$ and $\overline{u_{1}}=g_{1} a^{m}$ for some $m \in \mathbf{N}$. Furthermore, let $v_{2}, u_{2} \in M\left(S^{\prime}\right)$, with $v_{2} \neq u_{2}$, such that $v_{1}$ is a head of $v_{2}$, $u_{1}$ is a head of $u_{2}, \overline{v_{2}}=g_{2}$ and $\overline{u_{2}}=g_{2} a^{k}$ for some $k \in \mathbf{Z}$. We claim that $k>0$.

We prove this claim by induction on the $t$-length of $g_{2}$. The claim is clearly true if $l_{t}\left(g_{1}\right)=l_{t}\left(g_{2}\right)$ by the uniqueness of normal forms in $M(S)$, so assume $l_{t}\left(g_{1}\right)<l_{t}\left(g_{2}\right)$. Let $v^{\prime}$ be a head of $v_{2}$ and $u^{\prime}$ be a head of $u_{2}$, with $\overline{v^{\prime}}=g^{\prime}$, such that $l_{t}\left(g^{\prime}\right)=l_{t}\left(\overline{u^{\prime}}\right)=l_{t}\left(g_{2}\right)-1$ and $\overline{u^{\prime}}=g^{\prime} a^{n}$ where, by the inductive assumption, $n>0$. Then, by Lemma 2.4, $g_{2} \in\left\{g^{\prime} t a^{-1}, g^{\prime} t, g^{\prime} t a\right\}$, while $\overline{u_{2}} \in\left\{g^{\prime} a^{n} t a^{-1}, g^{\prime} a^{n} t, g^{\prime} a^{n} t a\right\}$, that is, $\overline{u_{2}} \in\left\{g^{\prime} t a^{2 n-1}, g^{\prime} t a^{2 n}, g^{\prime} t a^{2 n+1}\right\}$. But $g_{2} \neq \overline{u_{2}}$ since $M(S)$ gives unique normal forms, hence $\overline{u_{2}}=g_{2} a^{k}$, where $k>0$.

It follows from this that if $b_{1} w_{1}^{\prime}, b_{2} w_{2}^{\prime} \in M(S)$ such that $b_{1}, b_{2} \in\left\{a^{-1}, t, a\right\}$, $l_{t}\left(\overline{b_{1} w_{1}^{\prime}}\right)=l_{t}\left(\overline{b_{2} w_{2}^{\prime}}\right)$, and $b_{1}<b_{2}$, then $\overline{b_{1} w_{1}^{\prime} a^{k}}=\overline{b_{2} w_{2}^{\prime}}$ where $k>0$. The statement of the lemma now follows immediately by contradiction.

In order to show there exists a finite state automaton whose language is $M(S)$ we introduce a concept of 'types', which will enable us to construct a finite state automaton whose language is $M\left(S^{\prime}\right)$. Let $g \in S^{\prime}$ and let $g_{1}=g a^{-2}$, $g_{2}=g a^{-1}, g_{3}=g a, g_{4}=g a^{2}$. We define the 4-type of $g$ to be the 4-tuple $\left(e_{1}, e_{2}, e_{3}, e_{4}\right)$ where

$$
e_{i}= \begin{cases}l\left(g_{i}\right)-l(g) & \text { if } g_{i} \in S^{\prime} \\ 2 & \text { if } g_{i} \notin S^{\prime}\end{cases}
$$

So, for example, the 4-type of $t^{3} a$ is $(2,-1,0,1)$.

The following two lemmas make it clear why this definition of type is a useful one. 


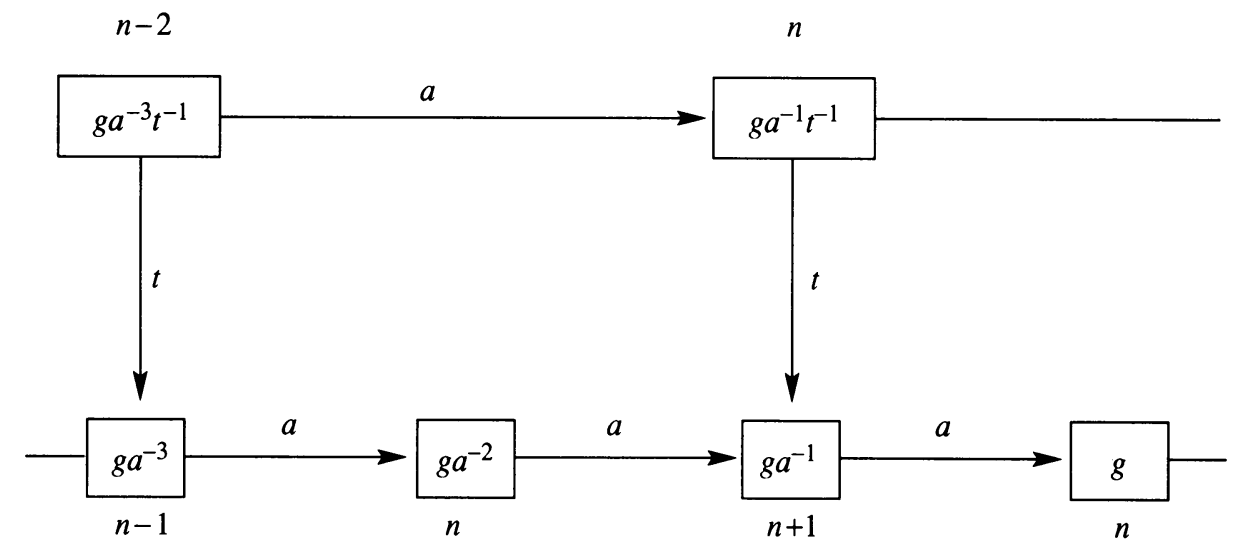

FIGURE 2

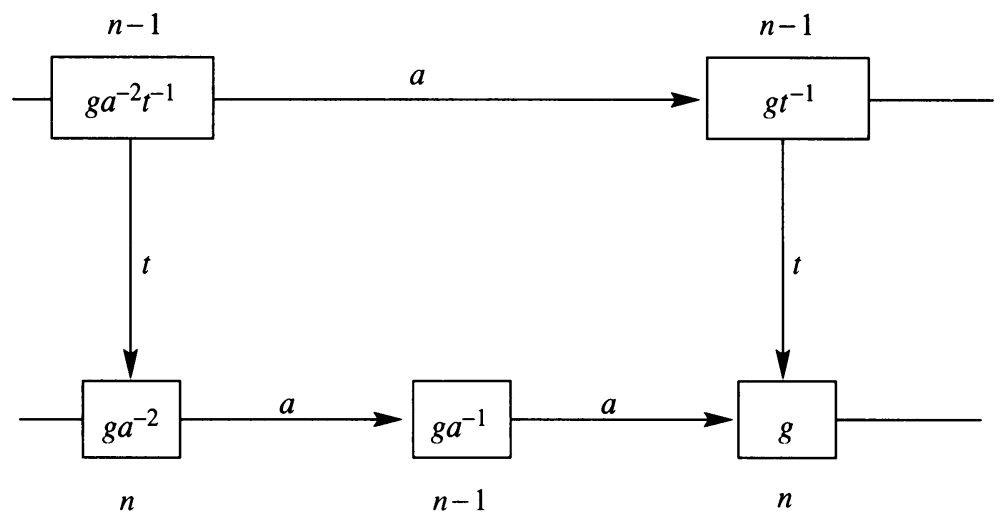

FIGURE 3

Lemma 2.7. Let $w \in M\left(S^{\prime}\right)$. The 4-type of $\bar{w}$ determines whether or not $w a^{-1}$, $w t$, and $w a$ are elements of $M\left(S^{\prime}\right)$.

Proof. Let $g=\bar{w}$ and $l(g)=n$. Note that $w \in M\left(S^{\prime}\right)$ implies $w t \in M\left(S^{\prime}\right)$, since no elements of $M\left(S^{\prime}\right)$ contain $a^{2}$ or $a^{-2}$ as a subword. For $w a^{-1}$ and $w a$, however, there are a couple of different cases to look at.

First we consider $w a^{-1}$. If $e_{2} \leq 0$ or if $e_{2}=2$ then clearly $w a^{-1} \notin M\left(S^{\prime}\right)$. So assume $e_{2}=1$. If $e_{1} \geq 1$ then $w a^{-1} \in M\left(S^{\prime}\right)$. Now let $e_{1}=0$, so $l(g)=l\left(g a^{-2}\right)=n$. There are two possibilities for the position of $g$ within $S^{\prime}$ : either $g a^{-1} t^{-1} \in S^{\prime}$ or $g a^{-2} t^{-1}, g t^{-1} \in S^{\prime}$. (These two possibilities correspond to Figures 2 and 3 respectively.) Assume $g a^{-1} t^{-1} \in S^{\prime}$. Then $l\left(g a^{-1} t^{-1}\right)=n$. But since $l\left(g a^{-2}\right)=n$ we must have $l\left(g a^{-3}\right)=n-1$ and hence $l\left(g a^{-3} t^{-1}\right)=n-2$ (see Figure 2). But this is a contradiction, since $g a^{-3} t^{-1}=g a^{-1} t^{-1} a^{-1}$.

Hence, $g a^{-2} t^{-1}, g t^{-1} \in S^{\prime}$ and the relevant section of the Cayley graph looks like Figure 3. In particular, note that $l\left(g a^{-2} t^{-1}\right)=l\left(g t^{-1}\right)=n-1$. Let $w_{1}, w_{2}$ be elements of $M\left(S^{\prime}\right)$ such that $w_{1}=g a^{-2} t^{-1}, w_{2}=g t^{-1}$. Clearly, 


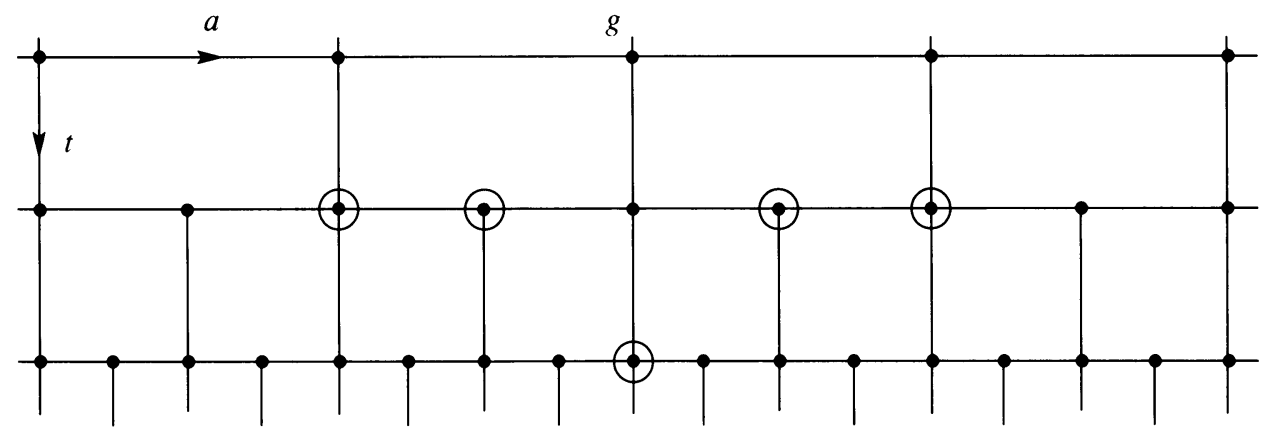

FIGURE 4

$\overline{w_{1} a}=\overline{w_{2}}$, hence $w_{1}<w_{2}$ by Lemma 2.6. So, $w_{1} t a<w_{2} t a^{-1}=w a^{-1}$, and consequently $w a^{-1} \notin M\left(S^{\prime}\right)$, since it is not lexicographically minimal.

Similarly, for $w a$ we have that if $e_{3} \leq 0$ or if $e_{3}=2$ then $w a \notin M\left(S^{\prime}\right)$, while if $e_{3}=1$ then $w a \in M\left(S^{\prime}\right)$ for all values of $e_{4}$.

Lemma 2.8. Let $w \in M\left(S^{\prime}\right)$, let $g=\bar{w}$, and let $b_{1}, b_{2} \in\left\{a^{-1}, t, a\right\}$. If $w b_{1} b_{2} \in M\left(S^{\prime}\right)$ then the 4-type of $\overline{w b_{1} b_{2}}$ is uniquely determined by the 4-type of $g$.

Proof. Consider the section of the Cayley graph of $S^{\prime}$ shown in Figure 4. Clearly the 4-type of $g$ allows us to determine the length (in terms of $l(g)$ ) of all the vertices shown in the diagram. In other words, we can determine the length of all $h \in S^{\prime}$ such that $g a^{-2} \succeq h$ and $h \preceq g a^{2}$. But by Lemmas 2.3 and $2.5, \overline{w b_{1} b_{2}}$ must be one of the five circled vertices, each of whose 4-type is determined by the 4-type of $g$.

Proof of Theorem 2.1. It is now a simple matter to show there is a finite state automaton whose language is $M\left(S^{\prime}\right)$ (as defined above). Let $g$ be any nonidentity element of $S^{\prime}$ and let $w$ be the corresponding word in $M\left(S^{\prime}\right)$. Write $w$ as $w_{1} b_{1}$ where $w_{1} \in M\left(S^{\prime}\right)$ and $b_{1} \in\left\{a^{-1}, t, a\right\}$. Define the state of $g$ to be the ordered pair $\left(T_{1}, T_{2}\right)$ where $T_{1}$ is the 4-type of $\overline{w_{1}}$ and $T_{2}$ is the 4-type of $g$. By Lemma 2.7 we can now determine exactly which words $w a^{-1}, w t$ and $w a$ are in $M\left(S^{\prime}\right)$ and for those that are in $M\left(S^{\prime}\right)$, we can determine the 4-types (and hence the states) of the corresponding elements, by Lemma 2.8 . Consequently, these states form an automaton whose language is $M\left(S^{\prime}\right)$, and since the number of different 4-types is clearly finite this automaton has a finite number of states. From this we can now produce an automaton whose language is $M(S)$. Observe that the elements of $S^{\prime} \backslash S$ can be characterized by their 4-types alone; in particular, they are precisely the elements of $S^{\prime}$ with 4-type $\left(e_{1}, e_{2}, 2,2\right)$. Hence, the elements of $M\left(S^{\prime}\right) \backslash M(S)$ are precisely characterized by the states $\left(T_{1}, T_{2}\right)$ for which $T_{2}$ is of the form $\left(e_{1}, e_{2}, 2,2\right)$. By changing all the states of this form from accepting states to failing states we obtain a finite state automaton whose language is $M(S)$. So $M(S)$ is regular, and thus, by Theorem 1.1, has rational growth. 
It is not difficult to construct an actual automaton whose language is $M(S)$ (as defined in the proof of the theorem) by applying the procedure in the above proof and removing redundant states. Figure 5 shows a suitable automaton $\mathscr{S}$, where the square vertices represent accepting states and the circular ones represent failing states. The automaton in the diagram has been superimposed upon a representation of the Cayley graph of $S$ in order to give an idea of what these minimal paths in $S$ look like geometrically.

An easy consequence of $M(S)$ being regular is the following proposition.

Proposition 2.9. The monoid $H=\left\langle a, a^{-1}, t \mid a a^{-1}=e, t a^{2}=a t\right\rangle$ has rational growth.

To prove this we will find a regular set of length-minimal normal forms for elements of $H$, and then apply Theorem 1.1. Let $T=\left\{g \in G_{2} \mid g / e\right\}$. We can repeat the arguments of Theorem 2.1 to show there is a regular set $M(T)$ of length-minimal normal forms for $T$ based on the lexicographical ordering $a<t<a^{-1}$. Again we can construct an actual finite state automaton, $\mathscr{T}$, whose language is $M(T)$. This automaton, which is basically just a mirror image of $\mathscr{S}$, is shown in Figure 6 on p. 146. We claim that the set

$$
M(H)=\left\{a^{k} w_{0} \mid k \in \mathbf{N}_{0}, w_{0} \in M(S)\right\} \cup\left\{a^{-l} w_{1} \mid l \in \mathbf{N}_{0}, w_{1} \in M(T)\right\}
$$

is a set of length-minimal normal forms for $H$.

In order to show this, the following definition is useful. Let $g \in H$ (or, more generally, $G_{2}$ ) such that $e \succeq g$. Then the a-reach of $g$ is the unique positive integer, $p$, for which $a^{p} \searrow g$. Similarly, if $g \prec e$, we define the $a^{-1}$-reach of $g$ to be value of $p$ for which $g \swarrow a^{-p}$.

The elements of $M(H)$ clearly provide unique normal forms for $H$. That this normal form for any $g \in H$ is length-minimal now follows by induction on the $a$-reach or $a^{-1}$-reach of $g$, where the inductive step is provided by the next lemma (which will also be used in $\S 3$ ).

Lemma 2.10. Let $g \in H$ such that $a \succeq g$, and let $w$ be a length-minimal word representing $g$. Then the first letter of $w$ is not $t$ or $a^{-1}$. Similarly, if $g \prec a^{-1}$, the first letter of a representative length-minimal word is not $a$ or $t$.

Proof. First consider the case for $a \succeq g$. Let $w_{0}$ be a word representing any $g_{0}$ such that $e \succeq g_{0}$. Assume the first letter of $w_{0}$ is $t$. It follows from Lemma 2.3 that $w_{0}$ contains no subword of the form $t a^{ \pm 2}$, hence $w_{0}$ can be written in the form $w_{0}=u_{1} u_{2} \cdots u_{k}$ where each $u_{i} \in\left\{t, t a^{-1}, t a\right\}$. But if $\overline{w_{1}} \prec g_{1}$ then $\overline{w_{1} u_{i}} \prec g_{1}$ for any $u_{i}$ in the above set, and so $g_{0} \prec a$.

If, on the other hand, we assume the first letter of $w_{0}$ is $a^{-1}$, then $w_{0}$ begins with the subword $a^{-j} a$ or $a^{-j} t$. The first of these subwords is not reduced and the second leads to a contradiction of the fact that $e \succeq g_{0}$, again by the above argument.

The case for $g \prec a^{-1}$ follows similarly.

It is easy to see that $M(H)$ is regular. Indeed, Figure 7 on p. 147 shows how to construct a nondeterministic finite-state automaton whose language is $M(H)$. So it follows from Theorem 1.1 that $H$ has rational growth. 


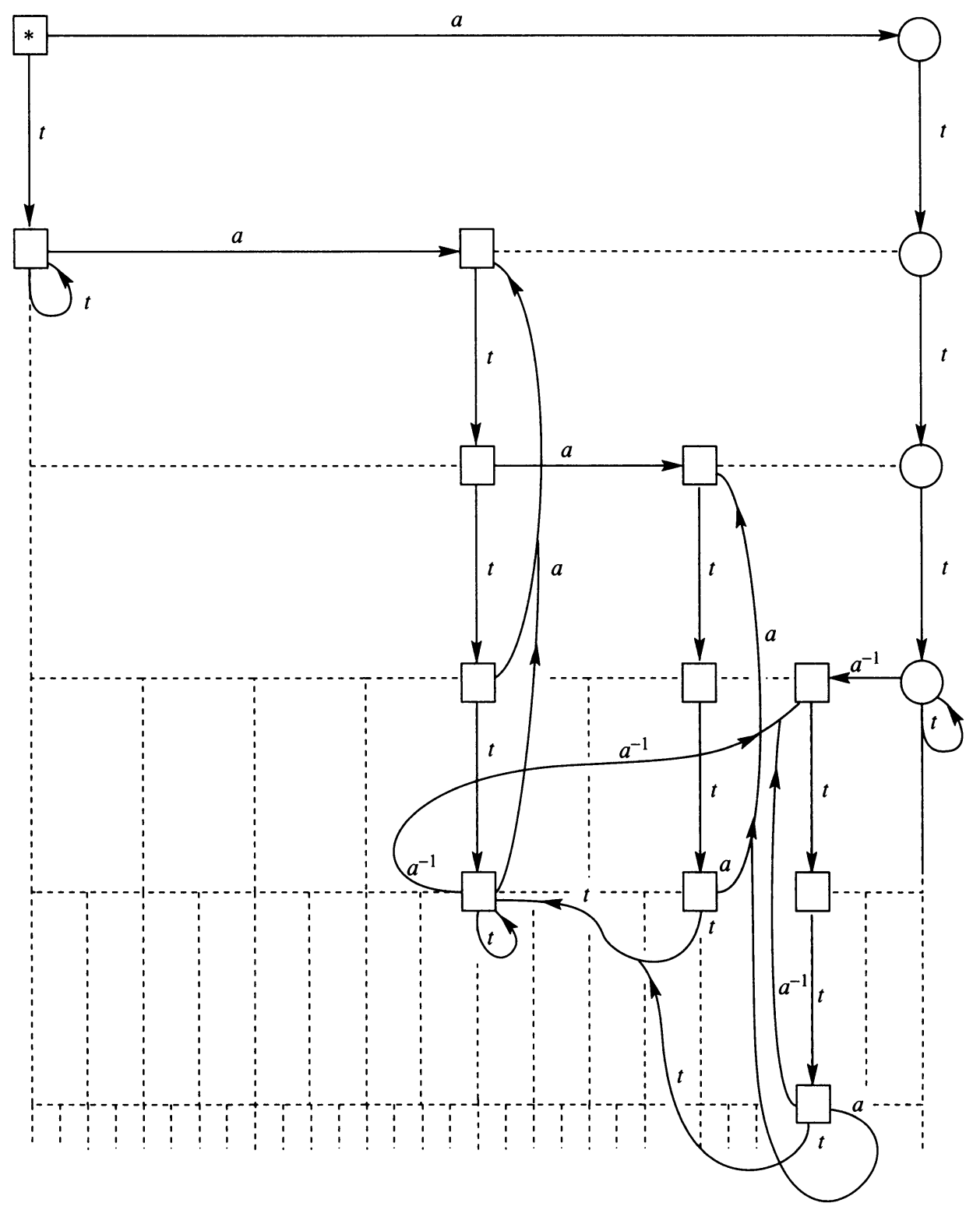

FIGURE 5. The automaton $\mathscr{S}$ 


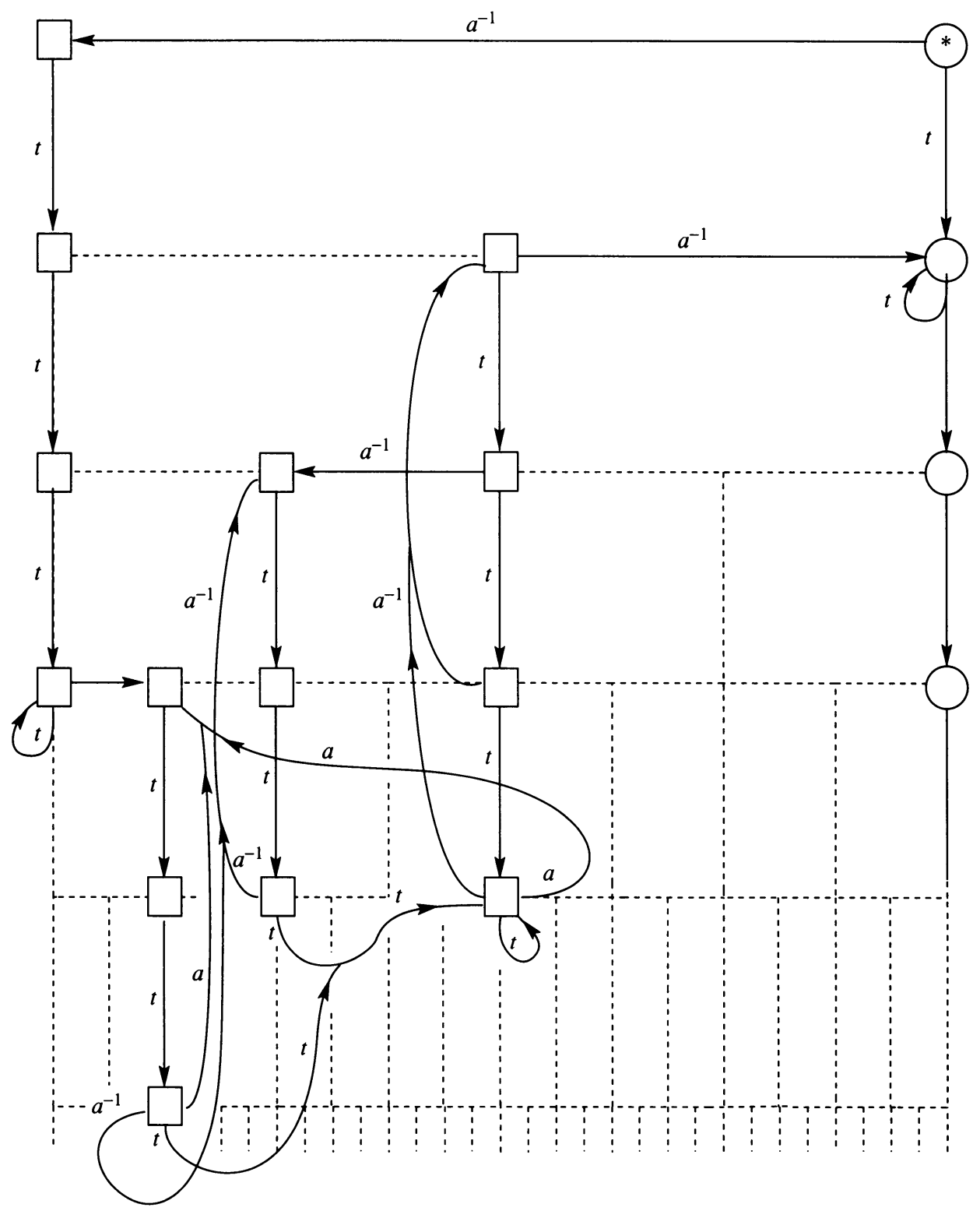

FIGURE 6. The automaton $\mathscr{T}$ 


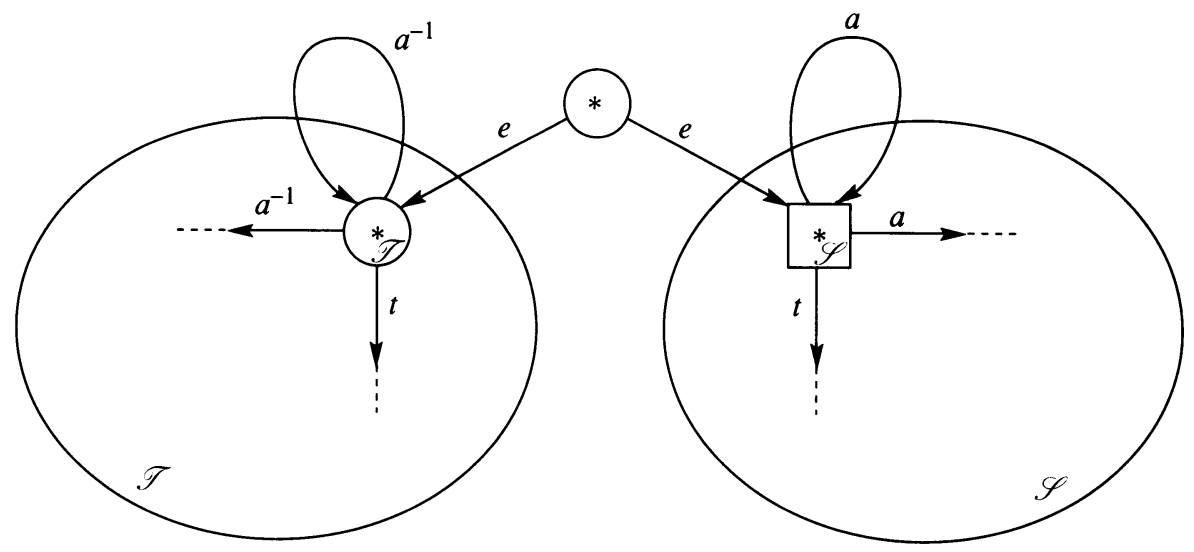

FIGURE 7. An automaton with language $M(H)$

3. THE GROWTH OF $G_{2}$

Before demonstrating that $G_{2}$ has rational growth, it will be useful to have the following elementary and standard result on rational generating functions.

Lemma 3.1. Let $\left(a_{n}\right)$ and $\left(b_{n}\right)$ be integer sequences such that

$$
\sum_{i=0}^{\infty} a_{i} x^{i} \text { and } \sum_{i=0}^{\infty} b_{i} x^{i}
$$

are rational functions of $x$. Then $\sum_{i=0}^{\infty} c_{i} x^{i}$ is also a rational function of $x$ if:

(i) $c_{i}=\left(a_{i} \pm b_{i+k}\right)$ for some $k \in N_{0}$;

(ii) $c_{i}=\sum_{j=0}^{i} a_{j}$;

(iii) $c_{i}=i a_{i}$; or

(iv) $c_{i}=\sum_{j=0}^{i} j a_{i-j}$.

Proof. Part (i) is trivial, while part (ii) follows immediately from the observation that $c_{i}-c_{i-1}=a_{i}$. For part (iii), let the $a_{i}$ 's satisfy the recurrence relation $\sum_{i=0}^{k} \alpha_{i} a_{n-i}$ for all $n$ sufficiently large. Then

$$
\sum_{i=0}^{k}(n-i+i) \alpha_{i} a_{n-i}=\sum_{i=0}^{k} \alpha_{i}\left(c_{n-i}+i a_{n-i}\right)=0 ;
$$

that is,

$$
\sum_{i=0}^{k} \alpha_{i} c_{n-i}=-\sum_{i=0}^{k} i \alpha_{i} a_{n-i}
$$

By (i) and (ii), the right-hand side of this equation satisfies some recurrence relation and hence the $c_{i}$ 's satisfy a recurrence relation. Finally, for part (iv), note that

$$
\sum_{j=0}^{i} j a_{i-j}=\sum_{k=0}^{i} \sum_{j=0}^{k} a_{j} .
$$

The result then follows by applying (ii) twice. 
We will now use the elements of $M(S)$ to construct length-minimal normal forms for $G_{2}$.

Theorem 3.2. $G_{2}$ has rational growth.

Proof. The strategy of the proof is to construct length-minimal normal forms for the elements of $G_{2}$ using the normal forms developed in the proof of Theorem 2.2 , and then show that these imply the group has rational growth. We divide the elements of $G_{2}$ into a number of different types, based on the partial ordering on $G_{2}$, and look at each of these cases individually.

Case 1. Let $g \in G$ such that $e \succeq g$. We will show that $g$ can be written uniquely in the form

(i) $a^{m_{0}} w$, where $m_{0} \in\{0,1,2,3\}$ and $w \in M(S)$, if $g \prec a^{4}$, or

(ii) $t^{-k} a^{m_{1}} w_{k}$, where $k \in \mathbf{N}, m_{1} \in\{2,3\}$, and $w_{k} \in M(S)$ such that $l_{t}\left(w_{k}\right) \geq k$, if $a^{4} \succeq g$,

and that these normal forms are length-minimal.

It is easy to see that this definition gives a unique normal form for each $g \in G_{2}$ such that $e \succeq g$. Recall that the $a$-reach of such a $g$ is the unique positive integer $p$ such that $a^{p} \searrow g$. Furthermore, if $g_{1}, g_{2} \in G_{2}$ with $g_{1} \succeq g_{2}$ we define the $a$-reach from $g_{1}$ to $g_{2}$ to be the $a$-reach of $g_{1}^{-1} g_{2}$. To show that the normal forms above are length-minimal we argue by induction on the $a$-reach of $g$.

Let $p$ be the $a$-reach of $g$, and let $g$ have a normal form corresponding to (i) or (ii) above. Arguing by contradiction, assume there is a length-minimal word $v$ with $\bar{v}=g$ such that $l(v)<l\left(a^{m_{0}} w\right)$ if $p<4$, or $l(v)<l\left(t^{-k} a^{m_{1}} w_{k}\right)$ if $p \geq 4$. By the proof of Lemma 2.10, we can assume that the first letter of $v$ is not $t$ or $a^{-1}$. (However note that in the second case there is an extra possibility that has to be considered, namely that $v$ could begin with a subword of the form $a^{-j} t^{-1}$. By applying Lemma 2.2 it is an easy exercise to show that $v$ begins with $a^{-j} t^{-1}$ only if there is another length-minimal word $v_{1}$ representing $g$, such that $v_{1}$ begins with the letter $t^{-1}$.) So we can assume $v$ begins with $t^{-1}$ or $a$. If $p=0$ then clearly no such $v$ exists since $w \in M(S)$. If $p=1$ then $t^{-1}$ must be a head of $v$. But $t^{-1} \searrow g$, so either $t^{-1} t$ or $t^{-1}$ at must be a head of $g$, which contradicts $v$ being length-minimal. Similarly, no suitable $v$ exists if $p=2$ or $p=3$.

Now let $p \geq 4$, and assume that the normal form is length-minimal for all elements with smaller $a$-reach. We consider in turn the two possibilities: $t^{-1}$ is a head of $v$, and $a$ is a head of $v$.

So assume $t^{-1}$ is a head of $v$, that is, there is a word $v_{0}$ such that $v=t^{-1} v_{0}$. Note that $t^{-1} \succ g$, but the $a$-reach from $t^{-1}$ to $g$ is smaller than that from $e$ to $g$. Hence, by induction, the word $t^{-(k-1)} a^{m_{1}} w_{k}$ represents a shortest path in the Cayley graph from $t^{-1}$ to $g$, contradicting the assumption that there is a shorter path $v_{0}$.

Alternatively, let $a$ be a head of $v$. Again we note that $a \succ g$, but the $a$ reach from $a$ to $g$ is smaller than that from $e$ to $g$. So $v$ must be of the form $t^{-k^{\prime}} a^{m_{1}^{\prime}} w_{k^{\prime}}$, where $k^{\prime} \in \mathbf{N}, m_{1}^{\prime} \in\{2,3\}$ and $w_{k^{\prime}} \in M(S)$ such that $l_{t}\left(w_{k^{\prime}}\right) \geq$ $k^{\prime}$. Let $l\left(t^{-k} a^{m_{1}} w_{k}\right)=q$. Since $l(v)<q$, we have $l\left(t^{-k^{\prime}} a^{m_{1}^{\prime}} w_{k^{\prime}}\right) \leq q-2$. Now consider the words $t^{-(k-1)} a^{m_{1}} w_{k}$ and $t^{-\left(k^{\prime}-1\right)} a^{m_{1}^{\prime}} w_{k^{\prime}}$. Both these words are length-minimal and $l\left(t^{-(k-1)} a^{m_{1}} w_{k}\right)=q-1$ while $l\left(t^{-\left(k^{\prime}-1\right)} a^{m_{1}^{\prime}} w_{k^{\prime}}\right) \leq$ 
$q-3$. We can write $w_{k^{\prime}}$ as $w_{1} w_{2}$ where $l_{t}\left(w_{1}\right)=k^{\prime}$. Consider the word $t^{-\left(k^{\prime}-1\right)} a^{m_{1}^{\prime}} w_{1} a w_{2}$. Clearly, by Lemma 2.2 ,

$$
\overline{t^{-k^{\prime}} a^{m_{1}^{\prime}} w_{1} a w_{2}}=\overline{a t^{-k^{\prime}} a^{m_{1}^{\prime}} w_{k^{\prime}}}=g
$$

hence

$$
\overline{t^{-\left(k^{\prime}-1\right)} a^{m_{1}^{\prime}} w_{1} a w_{2}}=\overline{t^{-(k-1)} a^{m_{1}} w_{k}}
$$

But $l\left(t^{-\left(k^{\prime}-1\right)} a^{m_{1}^{\prime}} w_{1} a w_{2}\right) \leq q-2$, contradicting the fact that $t^{-(k-1)} a^{m_{1}} w_{k}$ is length-minimal.

Case 2. Let $g \in G_{2}$ such that $g \prec e$. Here the length-minimal normal forms for $g$ are of the form

(i) $a^{-m_{0}} w$, where $m_{0} \in\{0,1,2,3\}$ and $w \in M(T)$, if $a^{-2} \succeq g$, or

(ii) $t^{-k} a^{-m_{1}} w_{k}$, where $k \in \mathbf{N}, m_{1} \in\{2,3\}$, and $w_{k} \in M(T)$ such that $l_{t}\left(w_{k}\right) \geq k$, if $g \prec a^{2}$.

The proof of this is identical in method to the proof in Case 1.

Case 3. Let $g \in G_{2}$ such that $g \succeq e$ or $e \prec g$, and in either case $g \neq a^{q}$ for any $q \in \mathbf{Z}$. From Cases 1 and 2 we already know a minimal path in the Cayley graph from $g$ to $e$, hence our length-minimal normal forms for this case are simply the formal inverses of the normal forms in Cases 1 and 2, for which $l_{t}\left(w_{k}\right)>k$.

Case 4. Let $g \in G_{2}$ such that $g$ and $e$ are incomparable under the partial ordering on $G_{2}$. First observe that in this case there is an obvious (non-lengthminimal) normal form for $g$ : namely, $g=t^{\alpha} a^{\beta} t^{-\gamma}$ where $\alpha, \gamma \in \mathbf{N}$ and $\beta$ is an odd integer. (Insisting that $\beta$ be odd is necessary for uniqueness since $a^{\beta}=t a^{2 \beta} t^{-1}$. It is equivalent to minimizing $\alpha$ and $\gamma$.) We will call $\alpha$ the $t$-height of $g$ and $\gamma$ the $t$-depth of $g$. Saying that $g$ and $e$ are incomparable under the partial ordering on $G_{2}$ is exactly equivalent to saying that the $t$-height and $t$-depth of $g$ are both strictly greater than zero. We will look in turn at two subcases based on the comparative size of $\alpha$ the $t$-height of $g$, and $\gamma$ the $t$-depth of $g$. These subcases are:

(1) $\alpha \geq \gamma$,

(2) $\alpha<\gamma$.

For the first subcase we will show that an appropriate length-minimal normal form for $g$ is $w t^{-\gamma}$ where $w$ is the normal form for $t^{\alpha} a^{\beta}$ given in Cases 1 and 2 . That this gives a unique normal form follows immediately from the uniqueness of $w$.

Define the elements $g[k, i] \in G_{2}$, where $k \in \mathbf{N}_{0}$ and $i \in \mathbf{Z}$, as $g[k, i]=$ $t^{k} a^{i}$ and let $w[k, i]$ be the length-minimal normal form for $g[k, i]$ given in Cases 1 and 2. We wish to show that the word $w[\alpha, \beta] t^{-\gamma}$ is lengthminimal. Clearly, $l\left(w[\alpha, \beta] t^{-\gamma}\right)=l(w[\gamma, 0])+l(w[\alpha, \beta])$. Now any path $v$ in the Cayley graph from $e$ to $g$ must pass through a vertex corresponding to an element of the form $g[\alpha, i]$ for some $i \in \mathbf{Z}$. If $v$ is lengthminimal then the two subpaths from $e$ to $g[\alpha, i]$ and from $g[\alpha, i]$ to $g$ are each length-minimal, and, by the symmetry of the Cayley graph $l(v)=$ $l(w[\alpha, i])+l(w[\gamma, j])$ where $j=\beta-i$. So what we now want to show is that the expression, $l(w[\gamma, i])+l(w[\alpha, \beta-i])$ is minimal when $i=0$. 


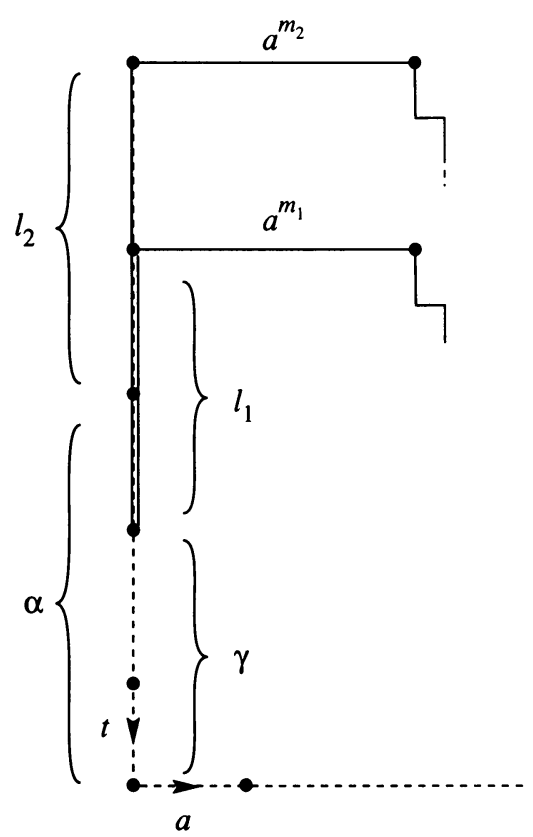

(a)

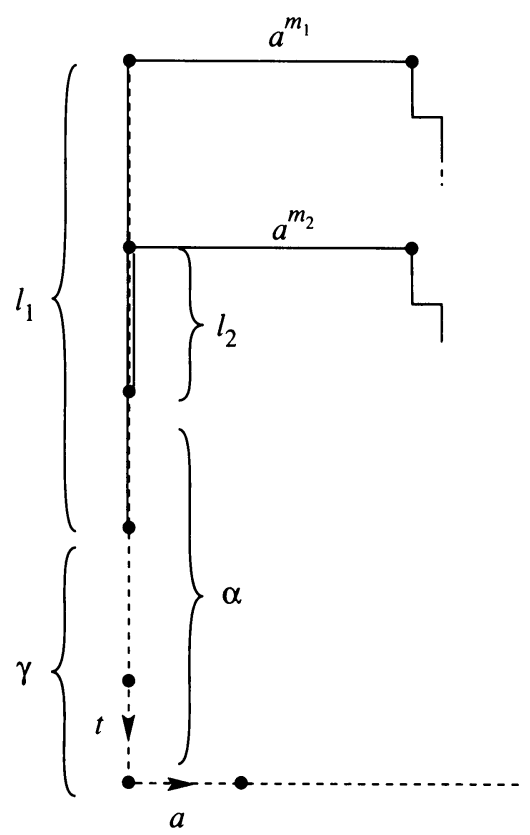

(b)

\section{FigURE 8}

By the normal forms given in Cases 1 and 2 there exist $l_{1}, l_{2} \in \mathbf{N}_{0}$ such that $t^{-l_{1}} a^{m_{1}} t$ is a head of $w[\gamma, i]$ and $t^{-l_{2}} a^{m_{2}} t$ is a head of $w[\alpha, \beta-i]$ for some $m_{1}, m_{2} \in \mathbf{Z}$. First consider the case where $\gamma+l_{1} \leq \alpha+l_{2}$, as illustrated in Figure 8(a). In this case we can write

$$
\begin{gathered}
w[\gamma, i]=t^{l_{1}} a^{m_{1}} t a^{\varepsilon_{1}} t a^{\varepsilon_{2}} \cdots t a^{\varepsilon_{k}}, \\
w[\alpha, \beta-i]=t^{-l_{2}} a^{m_{2}} w_{0} t a^{\delta_{1}} t a^{\delta_{2}} \cdots t a^{\delta_{k}}
\end{gathered}
$$

where $w_{0}$ is a word of $t$-height $\alpha+l_{2}-\gamma-l_{1}, k=\gamma+l_{1}$, and $\varepsilon_{i}, \delta_{i} \in$ $\{-1,0,1\}$. Now let $w_{\beta}=t^{-l_{2}} a^{m_{2}} w_{0} a^{m_{1}} t a^{\delta_{1}+\varepsilon_{1}} t a^{\delta_{2}+\varepsilon_{2}} \ldots t a^{\delta_{k}+\varepsilon_{k}}$. It is easy to see (by, for example, repeated applications of Lemma 2.2) that $\overline{w_{\beta}}=g[\alpha, \beta]$. Thus, by the construction of $w_{\beta}$ we have

$$
l(w[\gamma, i])+l(w[\alpha, \beta-i]) \geq l\left(t^{\gamma}\right)+l\left(w_{\beta}\right) \geq l(w[\gamma, 0])+l(w[\alpha, \beta]) .
$$

If on the other hand $\gamma+l_{1}>\alpha+l_{2}$, as in Figure 8(b), we can write

$$
\begin{gathered}
w[\gamma, i]=t^{l_{1}} a^{m_{1}} w_{0} t a^{\varepsilon_{1}} t a^{\varepsilon_{2}} \cdots t a^{\varepsilon_{k}}, \\
w[\alpha, \beta-i]=t^{-l_{2}} a^{m_{2}} t a^{\delta_{1}} t a^{\delta_{2}} \cdots t a^{\delta_{k}}
\end{gathered}
$$

where $w_{0}$ is a word of $t$-height $\gamma+l_{1}-\alpha-l_{2}, k=\alpha+l_{2}$, and $\varepsilon_{j}, \delta_{j} \in$ $\{-1,0,1\}$. This time, let $w_{\beta}=t^{-\left(\gamma+l_{1}-\alpha\right)} a^{m_{1}} w_{0} a^{m_{2}} t a^{\delta_{1}+\varepsilon_{1}} t a^{\delta_{2}+\varepsilon_{2}} \cdots t a^{\delta_{k}+\varepsilon_{k}}$. As before, we again have that the minimum value of $l(w[\gamma, i])+l(w[\alpha, \beta-i])$ occurs when $i=0$. Thus we have demonstrated for the first subcase that the given normal forms for $g$ are length-minimal. 


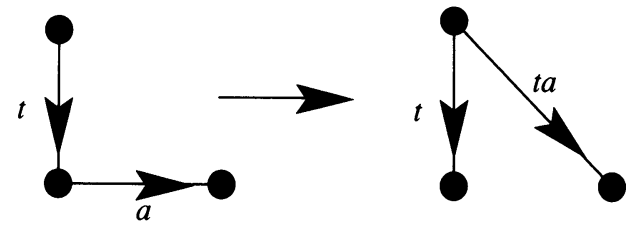

and

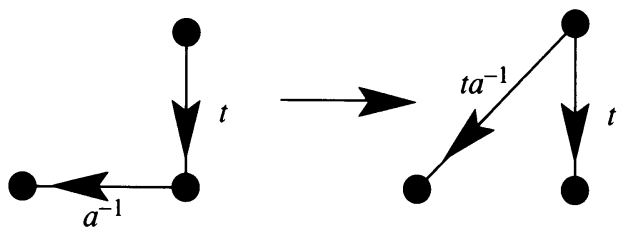

FIGURE 9

For the second subcase, where $\alpha<\gamma$, note that the first subcase already gives us a unique minimal path in the Cayley graph from $g$ to $e$. So a suitable set of length-minimal normal forms here is the set of inverses of length-minimal normal forms from subcase (1) where $\alpha>\gamma$. This concludes Case 4.

Cases 1 to 4 now give us a complete set of length-minimal normal forms for $G_{2}$, which we will call $C$. We will denote by $C(j)$ the set of normal forms given by Case $\mathrm{j}$, and we will let $c(j)_{n}:=\operatorname{card}\{w \in C(j) \mid l(w)=n\}$. To complete the proof of the theorem we will show that each $C(j)$ has rational growth, in other words that, for each $j, \sum_{0}^{\infty} c(j)_{i} x^{i}$ is a rational function of $x$. Again we proceed on a case by case basis, considering each of the above cases in turn.

Case 1. Define the integer sequences $\left(a_{n}\right),\left(b_{n}\right)$ and $\left(b_{n}^{\prime}\right)$ as follows

$$
\begin{aligned}
& a_{n}=\operatorname{card}\{w \in M(S) \mid l(w)=n\}, \\
& b_{n}=\operatorname{card}\left\{w \in M(S) \mid l_{t}(w)=n\right\}, \\
& b_{n}^{\prime}=\operatorname{card}\left\{w \in M(S) \mid l_{t}(w) \leq n\right\} .
\end{aligned}
$$

From Section 2 we know that $\sum_{0}^{\infty} a_{i} x^{i}$ is a rational function of $x$. To see that $\sum_{0}^{\infty} b_{i} x^{i}$ is rational, consider again the finite state automaton $\mathscr{S}$ in Figure 5 whose language is $M(S)$. We can modify $\mathscr{S}$ as follows:

(i) For each set of states $s_{1}, s_{2}$ and $s_{3}$ in $\mathscr{S}$, such that there is an edge from $s_{1}$ to $s_{2}$ labelled $t$, a loop at $s_{2}$ labelled $t$ and an edge from $s_{2}$ to $s_{3}$ labelled $a$ (or $a^{-1}$ ), relabel the edge from $s_{2}$ to $s_{3}$ as $t a$ (or $t a^{-1}$ respectively) and add an edge from $s_{1}$ to $s_{3}$ also labelled $t a$ (or $t a^{-1}$ respectively).

(ii) For each set of states $s_{1}, s_{2}$ and $s_{3}$ in $\mathscr{S}$ (with $s_{1}$ and $s_{2}$ not necessarily distinct), such that there is an edge from $s_{1}$ to $s_{2}$ labelled $t$, an edge from $s_{2}$ to $s_{3}$ labelled $a$ (or $a^{-1}$ ) and no loop at $s_{2}$, remove the edge from $s_{2}$ to $s_{3}$ and add an edge from $s_{1}$ to $s_{3}$ labelled $t a$ (or $t a^{-1}$ respectively). See Figure 9.

(iii) Finally, remove the state in the top right-hand corner and its adjacent edges, and add an edge from the initial state to the state below the removed state, labelled at . 
This new automaton, say $\mathscr{S}_{t}$, has the same language as $\mathscr{S}$ except that it expresses the normal forms in terms of the subwords $t$, at, ta and $t a^{-1}$. Hence the length associated with any $w$ in the language of $\mathscr{S}_{t}$ is the $t$-length of $w$. It now follows from the proof of Theorem 1.1 that $\sum_{0}^{\infty} b_{i} x^{i}$ is rational. Also, it follows from Lemma 3.1 that $\sum_{0}^{\infty} b_{i}{ }_{i} x^{i}$ is rational.

Now let $v \in C(1)$. Then $v$ must have one of the following forms:

(1) $v=w$ where $w \in M(S)$;

(2) $v=a w$ where $w \in M(S)$;

(3) $v=t^{-k} a^{2} w_{k}$ where $k \in \mathbf{N}_{0}$, and $w_{k} \in M(S)$ such that $l_{t}\left(w_{k}\right) \geq k$; or

(4) $v=t^{-k} a^{3} w_{k}$ where $k \in \mathbf{N}_{0}$, and $w_{k} \in M(S)$ such that $l_{t}\left(w_{k}\right) \geq k$. Calculating the number of elements in $C(1)$ of length $n$ for each form of $v$ we have:

$$
\begin{aligned}
\operatorname{card} & \left\{t^{-k} a^{2} w \mid w \in M(S), l\left(t^{-k} a^{2} w\right)=n, \text { and } l_{t}(w) \geq k\right\} \\
& =\operatorname{card}\left\{w \in M(S) \mid l(w)=n-k-2 \text { and } l_{t}(w) \geq k\right\} \\
& =\sum_{k=0}^{n}[\operatorname{card}\{w \in M(S) \mid l(w)=n-k-2\} \\
& =\sum_{k=0}^{n}\left[a_{n-k-2}-b_{k-1}^{\prime}\right] .
\end{aligned}
$$

$$
\begin{gathered}
\operatorname{card}\left\{t^{-k} a^{3} w \mid w \in M(S), l\left(t^{-k} a^{3} w\right)=n, \text { and } l_{t}(w) \geq k\right\} \\
=\sum_{k=0}^{n}\left[a_{n-k-3}-b_{k-1}^{\prime}\right] \text { (by the same argument). }
\end{gathered}
$$

Hence

$$
c(1)_{n}=a_{n}+a_{n-1}+\sum_{k=0}^{n}\left(a_{n-k-2}-b_{k-1}^{\prime}+a_{n-k-3}-b_{k-1}^{\prime}\right),
$$

and so $\sum_{0}^{\infty} c(1)_{i} x^{i}$ is a rational function of $x$ by Lemma 3.1.

Case 2. As in the previous case we define integer sequences $\left(A_{n}\right),\left(B_{n}\right)$ and $\left(B_{n}^{\prime}\right)$ as follows:

$$
\begin{aligned}
& A_{n}=\operatorname{card}\{w \in M(T) \mid l(w)=n\} \\
& B_{n}=\operatorname{card}\left\{w \in M(T) \mid l_{t}(w)=n\right\} \\
& B_{n}^{\prime}=\operatorname{card}\left\{w \in M(T) \mid l_{t}(w) \leq n\right\} .
\end{aligned}
$$

Again by a similar argument we deduce that

$$
c(2)_{n}=A_{n}+A_{n-1}+\sum_{k=0}^{n}\left(A_{n-k-2}-B_{k-1}^{\prime}+A_{n-k-3}-B_{k-1}^{\prime}\right),
$$

and hence that $\sum_{0}^{\infty} c(2)_{i} x^{i}$ is rational. 
Case 3. The normal forms for this case are simply the inverses of the normal forms for Cases 1 and 2 with the added condition that $v \neq a^{q}$ for any $q \in \mathbf{Z}$. It immediately follows that $c(3)_{0}=0, c(3)_{1}=1$ and

$$
\begin{aligned}
c(3)_{n}= & a_{n}+a_{n-1}+\sum_{k=0}^{n}\left(a_{n-k-2}-b_{k}^{\prime}\right)+\sum_{k=0}^{n}\left(a_{n-k-3}-b_{k}^{\prime}\right) \\
& +A_{n}+A_{n-1}+\sum_{k=0}^{n}\left(A_{n-k-2}-B_{k}^{\prime}\right)+\sum_{k=0}^{n}\left(A_{n-k-3}-B_{k}^{\prime}\right)
\end{aligned}
$$

for $n>2$.

Case 4. Let $M\left(S^{*}\right)=\left\{w \in M(S) \mid a\right.$ or $a^{-1}$ is a tail of $\left.w\right\}$ and $M\left(T^{*}\right)=\{w \in$ $M(T) \mid a$ or $a^{-1}$ is a tail of $\left.w\right\}$. If we modify the finite state automaton $\mathscr{S}$ by changing every state with an edge labelled $t$ pointing to it to a failing state, we obtain a finite state automaton whose language is $M\left(S^{*}\right)$. Hence $M\left(S^{*}\right)$ and, similarly, $M\left(T^{*}\right)$ are regular, and so if

$$
\alpha_{n}=\operatorname{card}\left\{w \in M\left(S^{*}\right) \cup M\left(T^{*}\right) \mid l(w)=n\right\},
$$

for all $n \geq 0$, then $\sum_{0}^{\infty} \alpha_{i} x^{i}$ is a rational function of $x$. Also, if $\boldsymbol{\beta}^{\prime}{ }_{n}=$ $\operatorname{card}\left\{w \in M\left(S^{*}\right) \cup M\left(T^{*}\right) \mid l_{t}(w) \leq n\right\}$, then a similar argument to that used in Case 1 tells us that $\sum_{0}^{\infty}{\beta^{\prime}}_{i} x^{i}$ is rational.

For subcase (1), the normal forms are of the form:

(i) $a^{m_{0}} w t^{-l} \quad$ where $\quad w \in M\left(S^{*}\right) \cup M\left(T^{*}\right)$,

$$
m_{0} \in \begin{cases}\{0,1\} & \text { if } w \in M\left(S^{*}\right) \\ \{0,-1\} & \text { if } w \in M\left(T^{*}\right)\end{cases}
$$

and $1 \leq l \leq l_{t}(w)$; or

(ii) $t^{-k} a^{m_{1}} w t^{-l}$ where $w \in M\left(S^{*}\right) \cup M\left(T^{*}\right)$,

$$
m_{1} \in \begin{cases}\{2,3\} & \text { if } w \in M\left(S^{*}\right) \\ \{-2,-3\} & \text { if } w \in M\left(T^{*}\right)\end{cases}
$$

and $l \in \mathbf{N}, k \in \mathbf{N}_{0}$ such that $k+l \leq l_{t}(w)$.

Again, via a similar argument to that used in Case 1, we calculate that the number of elements of length $n$ for the first possibility is

$$
\sum_{l=1}^{n}\left(\alpha_{n-l}+\beta_{l-1}^{\prime}\right)+\sum_{l=1}^{n}\left(\alpha_{n-l-1}+\beta_{l-1}^{\prime}\right) .
$$

For the second possibility, if $m=l+k$ then there are $m-1$ distinct pairs $(l, k)$ with $l \in \mathbf{N}$ and $k \in \mathbf{N}_{0}$. It follows that the number of elements of length $n$ for the second possibility is

$$
\sum_{m=1}^{n}(m-1)\left[\left(\alpha_{n-m-2}-\beta_{k-1}^{\prime}\right)+\left(\alpha_{n-m-3}-\beta_{k-1}^{\prime}\right)\right] .
$$

Now $c(4.1)_{n}$ is the sum of these two expressions, and so it follows from Lemma 3.1 that $\sum_{0}^{\infty} c(4.1)_{i} x^{i}$ is rational. Similarly, $\sum_{0}^{\infty} c(4.2)_{i} x^{i}$ is rational, completing Case 4. 
Finally, if $c_{n}=\operatorname{card}\{w \in C \mid l(w)=n\}$, then $c_{n}=c(1)_{n}+c(2)_{n}+c(3)_{n}+$ $c(4.1)_{n}+c(4.2)_{n}$ and hence $\sum_{0}^{\infty} c_{i} x^{i}$ is rational.

Remarks. (1) In principle it should be possible to calculate the exact growth function $\Gamma\left(G_{2},\left\{a, t, a^{-1}, t\right\}\right)(x)$. For any sequence $\left(d_{n}\right)$ defined by $d_{n}=$ $\operatorname{card}\{w \in M \mid l(w)=n\}$ where $M$ is the language of a finite state automaton, a closed form for the expression $\sum_{0}^{\infty} d_{i} x^{i}$ can be determined by considering the adjacency matrix of the automaton. An example of this is given in [3], and an efficient computer algorithm for generating this closed form is discussed in [8]. Basically, the proof of the above theorem shows how to express the growth of $G_{2}$ in terms of rational functions which can be exactly determined via finite state automata. In practice, however, calculating the growth of $G_{2}$ by this method would be rather complicated and tedious.

(2) It is not hard to see that Theorem 3.2 can be generalized to all BaumslagSolitar groups of the form $G_{n}=\left\langle a, t \mid t a^{n}=a t\right\rangle$. To prove this we define partial orderings exactly as in the case of $G_{2}$ and show that the obvious analogue to Theorem 2.1 holds. Again every element of $G_{n}$ can be written uniquely in the form $a^{\beta} t^{-\gamma}$ where $\beta \in \mathbf{Z}, \gamma \in \mathbf{N}$, or $t^{\alpha} a^{\beta} t^{-\gamma}$ where $\alpha, \gamma \in \mathbf{N}_{0}$ and $\beta \in \mathbf{Z}$ such that $\beta \neq 0(\bmod n)$, allowing us to construct length-minimal normal forms for the elements of $G_{n}$ in terms of words in $M(S)$. A similar combinatorial argument to that used above then shows that $G_{n}$ has rational growth. In essence, the proof of this result is no more difficult than the argument for $G_{2}$, but the details are somewhat more technical. An outline of some of these details appears in [4].

Note, however, that there is not an immediately obvious way to generalize the theorem to the Baumslag-Solitar groups $G_{p, q}=\left\langle a, t \mid t a^{p}=a^{q} t\right\rangle$ where $p \neq q$ and $p, q \geq 2$. The problem is that for these groups normal forms are considerably more complicated. Nevertheless, it seems reasonable to conjecture that these groups also have rational growth, and it is possible that a variation of the methods developed in this paper can be used to confirm this conjecture.

\section{REFERENCES}

1. G. Baumslag and D. Solitar, Some two-generator one-relator non-Hopfian groups, Bull. Amer. Math. Soc. 68 (1962), 199-201.

2. G. Baumslag, S. M. Gersten, M. Shapiro, and H. Short, Automatic groups and amalgams, MSRI preprint.

3. M. Brazil, Monoid growth functions for braid groups, Internat. J. Algebra and Comput. 1 (1991), 201-205.

4. _ Groups with rational growth, Ph.D. Thesis, La Trobe University, 1992.

5. M. Edjvet and D. L. Johnson, The growth of certain amalgamated free products and $\mathrm{HNN}$ extensions, J. Austral. Math. Soc. (to appear).

6. D. B. A. Epstein, J. W. Cannon, D. F. Holt, M. S. Patterson and W. P. Thurston, Word processing in groups, Jones and Bartlett, Boston, Mass., 1992.

7. E. Ghyst and P. de la Harpe (eds.), Sur les groupes hyperboliques d'apres Mikhael Gromov, Birkhäuser, Boston, Mass., 1990.

8. U. Zwick, Computing growth functions, University of Warwick, preprint.

Department of Mathematics, La Trobe University, Bundoora, Victoria 3083, AusTRALIA

Current address: Department of Mathematics, University of Melbourne, Parkville, Victoria 3052 Australia

E-mail address: brazil@mundoe.maths.mu.oz.au 